\title{
Au Mexique, l'état a interdit à la religion l'accès à la propriété des médias
}

Armando Zacarías Castillo

\section{(2) OpenEdition}

1 Journals

Édition électronique

URL : http://journals.openedition.org/communicationorganisation/1838

DOI : 10.4000/communicationorganisation. 1838

ISSN : $1775-3546$

Éditeur

Presses universitaires de Bordeaux

Édition imprimée

Date de publication : 1 mai 1996

ISSN : 1168-5549

Référence électronique

Armando Zacarías Castillo, « Au Mexique, l'état a interdit à la religion l'accès à la propriété des médias », Communication et organisation [En ligne], 9| 1996, mis en ligne le 26 mars 2012, consulté le 30 avril 2019. URL : http://journals.openedition.org/communicationorganisation/1838 ; DOI :

10.4000/communicationorganisation.1838

Ce document a été généré automatiquement le 30 avril 2019

(c) Presses universitaires de Bordeaux 


\title{
Au Mexique, l'état a interdit à la religion l'accès à la propriété des médias
}

\author{
Armando Zacarías Castillo
}

1 Les moyens de communication de masse sont devenus aujourd'hui des instruments essentiels à la diffusion des idées et des valeurs. Dans plusieurs pays, les autorités religieuses ont compris que pour avoir un contrôle sur la diffusion de leur message, le meilleur moyen était de devenir propriétaire des médias. Or, au Mexique, la propriété des médias est interdite à toute religion. Le texte qui suit explique les circonstances qui ont amené le pouvoir Politique à adopter une telle mesure et décrit les pressions exercées par l'Église catholique pour renverser cette situa-don. Il présente enfin la façon dont l'Église catholique a contourné en partie l'interdit de posséder des médias par une occupation quasi totale du champ de l'éducation universitaire à la communication sociale.

$2 \mathrm{Au}$ Mexique, la religion catholique a toujours dominé la scène religieuse et un pourcentage assez élevé de Mexicains se déclarent croyants. Bien que la présence de ${ }^{c}$ ultes non catholiques se soit manifestée tout au long de l'histoire mexicaine, et certains de ceux-ci ont même pris Une certaine ampleur au cours des dernières années, l'impact culturel qu'ils exercent dans le contexte général du pays s'est dessiné à l'ombre de la religion catholique.

3 Par ailleurs, la constitution actuelle du Mexique a imposé à l'État une structure laïque, encadrant et balisant la présence et le pouvoir que détenait alors l'Église catholique. C'est ainsi que l'accès à la propriété des moyens électroniques de communication a été interdit aux cultes religieux mexicains. Cette circonstance a provoqué divers affrontements entre l'Église et l'État jusqu'à aujourd'hui. La situation n'a pas été modifiée de façon substantielle malgré les progrès qu'on a commencé à observer en matière d'administration des moyens électroniques dans différentes parties du territoire mexicain.

4 En 1990, au moment où des approches politiques entre l'Église et l'État se multipliaient dans un essai sérieux pour rétablir des rapports plus harmonieux, les bureaux de communication sociale des archevêchés mexicains prenaient conscience qu'ils devaient 
vivre avec les instruments de cette fin de siècle. Le père Felipe de Jesús Leal Galván, responsable de la communication sociale de l'archevêché de Monterrey précisait par exemple que :

«loin de croire que l'Église (catholique) devrait rester en marge ou essayer de s'isoler [de la technologie des communications]... les prêtres ont compris que l'Église devait être partenaire de ces technologies, se mettant ainsi au rythme des expériences de l'humanité, en essayant de les comprendre et de les interpréter à la lumière de la foi »(El Norte, 27/05/90).

5 La réflexion se poursuit toujours et l'Église s'ouvre à l'utilisation des nouvelles ressources technologiques telles que l'informatique, les satellites et les moyens électroniques de communication de masse. En même temps s'accentue le besoin de proposer un réajustement à la réglementation pour permettre à l'église d'avoir une plus grande participation dans tous les moyens de communication de masse (El Norte, ibid).

6 L'accès de l'Église aux mass-médias s'est trouvé brimé par l'État qui craignait qu'une institution aussi importante que l'Église catholique puisse acquérir plus de pouvoir en administrant ou en possédant un outil significatif de diffusion. Le responsable de la communication sociale de la Conférence Épiscopale Mexicaine a déclaré en ce sens :

«nous sommes très gênés parce que chaque fois que nous assistons à quelque rencontre internationale pour informer nos confrères sur ce que l'Église catholique fait en matière de mass-médias au Mexique, nous sommes obligés de dire : rien [...] La législation nous empêche d'avoir un accès direct à la presse, à la radio ou à la télévision [...] Ici ce n'est pas comme dans le reste de l'Amérique latine, nous avons des lois qui limitent nos droits humains les plus élémentaires » (El Norte, 19/12/90).

Le processus de rétablisement des rapports entre l'Église et l'État a pris un certain essor lors de l'accès à la présidence de Carlos Salinas (1988-1994). Celui-ci, dans un acte totalement inattendu, a invité en 1988 les principaux membres de la hiérarchie catholique mexicaine à la cérémonie officielle de son entrée en fonction. Le rétablissement des rapports entre les deux institutions se mettait en marche. Néanmoins les Églises, et particulièrement la catholique, ne trouvèrent pas la même ouverture en ce qui a trait à leur incorporation dans le cadre de la reconnaissance juridique, de telle sorte que cette reconnaissance se faisait toujours attendre. L'ouverture politique évoluait plus rapidement que l'ouverture juridique.

Dans ce contexte, la réclamation la plus constante de l'Église catholique fut celle de sa participation comme acteur-propriétaire des mass-médias. La Conférence Épiscopale Mexicaine demanda des réformes législatives pour que l'Église catholique du pays ait accès aux mass-médias, qu'elle qualifiait de " chaires modernes ».

9 L'éventuelle possibilité que l'Église catholique puisse obtenir enfin une concession de l'État pour devenir propriétaire d'un moyen électronique de communication a paru vraisemblable au début des années 90 . Cette ouverture n'a pas manqué de créer un certain espoir et plus précisement lors de la Conférence Épiscopale où avait été soulevé le besoin d'une meilleure "présence et formation de ses propres communicateurs, pour accomplir le but tracé par Jésus-Christ dans l'Évangile [...] L'homme qui voudrait échapper à la communication ne serait rien, il tomberait dans le vide " (El Norte, 09/08/91).

10 L'Église mexicaine souffrait en effet d'un véritable décalage face aux autres pays de l'Amérique latine où les discussions sur la communication sociale de l'Église avaient 
depuis longtemps dépassé la simple propriété des médias et traitaient de problèmes beaucoup plus spécifiques.

11 Pour remédier à cette situation, l'Église développa une approche régionale et locale et ses actions au niveau des communautés de base ont stimulé le développement de techniques et de stratégies de communication communautaire qui se sont révélées avec le temps fort efficaces. Cependant, dans une certaine mesure, l'Église ne jouait de cette façon qu'un rôle d'animation et de conseil. Ainsi, paradoxalement, alors que la communication de masse devenait l'outil de diffusion par excellence, pour l'Église, le recours à l'approche communautaire ne cessait de croître et constituait une parade à son exclusion des grands médias de masse.

Cette exclusion n'était pas innocente et, à travers elle, se dessinait une vieille lutte de pouvoir entre l'État et l'Église au Mexique, l'État craignant le retour en force de l'Église si elle venait à posséder ses propres outils de communication de masse au niveau national.

Le 17 décembre 1992, 460 députés ont voté en faveur du rétablissement des rapports entre l'Église et l'État, ne rencontrant qu'une très faible résistance de 22 députés qui ont voté contre l'adoption de cette mesure.

Le lendemain de l'approbation de l'amendement constitutionnel, l'Église catholique annonçait son intention d'acheter une chaîne de télévision. Dans les faits, « la hiérarchie catholique ne disposait pas des ressources nécessaires pour acheter une chaîne de T.V. ou même une station de radio » (El Norte, 18/12/91).

Déjà en 1991, lorsque la proposition de l'amendement constitutionnel fut connu, et qu'on eut l'impression qu'il s'agissait d'une véritable ouverture, on assista à une double négociation politique. D'une part, les négociations au sommet se révélaient très actives; d'autre part, aux niveaux intermédiaires, elles stagnaient. Dans de telles conditions, même après que les amendements constitutionnels eurent été votés en 1992, les Églises ont continué à dénoncer leurs conditions d'application. La Conférence Épiscopale Mexicaine déplorait «l'ouverture insuffisante " que l'État octroyait à l'Église en matière de communication et plus spécifiquement la Conférence signalait le "peu d'espaces qui lui étaient réservés dans le domaine éducatif et surtout le fait de lui refuser le droit de s'exprimer sur des sujets non religieux, ainsi que d'accéder à la propriété de moyens de communication de masse ».

\section{L'interdiction légale de participer à la vie Publique}

Au Mexique, strictement parlant, il serait difficile d'affirmer que le politique manifeste une attitude anti-religieuse. Il faut toutefois préciser que la Constitution mexicaine actuellement en vigueur a imposé dès l'origine de fortes limitations d'action à l'Église catholique, seule religion autorisée sous le régime colonial précédent, en limitant les cultes religieux à une activité purement privée. Ce qu'on peut signaler, c'est une attitude plutôt anticléricale qui a prédominé au fil des ans tout au long du XXe siècle. À l'époque de la formation de l'État central, une forte concurrence politique a opposé ces deux acteurs Pour le contrôle de la vie civile. Roberto Blancarte signale que :

« la dispute pour les masses devient alors la question centrale des rapports entre l'Église catholique et l'État post-révolutionnaire. La hiérarchie religieuse ainsi que les dirigeants du régime sont conscients que l'orientation générale de la société sera à la disposition de ceux qui contrôlent l'organisation et l'éducation du peuple » (Blancarte, 1993 :157). 
17 L'enjeu était donc politique et non pas religieux, ce qui explique ce paradoxe: l'intolérance de l'État à l'égard du clergé et la tolérance face à la pratique religieuse comme telle. années 1926 à 1940, et plus précisément encore les années 1926 à 1929, appelées la Guerra Cristera. Les affrontements entre l'Église et l'État se sont alors durcis et se sont dégradés jusqu'à la violence armée. Puis, l'État a commencé à gagner du terrain par sa présence institutionnelle et en raison de l'attitude du clergé qui a adopté de plus en plus des positions politiques que la population n'acceptait pas. Les gens ont commencé à reprocher ses actions au clergé et petit à petit l'image de l'Église s'est détériorée auprès du peuple.

19 La tentative de récupération du terrain perdu face à l'État a poussé l'Église à mettre en marche diverses stratégies pour se rapprocher de la population. Toutefois, malgré l'essai d'assainissement des relations entre l'Église et les citoyens, on assista à la naissance d'une attitude particulière des Mexicains, qui consistait en un fort attachement culturel envers la religion et, en même temps, en une forte critique contre la participation du clergé dans les affaires politiques ou de l'État. Dès les années 50, l'État se consolide autour d'un parti central qui incorpore les forces triomphantes de la Révolution. L'État et le parti officiel sont assez fréquemment confondus et toute la vie politique a comme centre deux institutions: le président (le «présidentialisme») et le Parti Révolutionnaire Institutionnel (PRI), corporatiste.

À cette époque, la société en général changeait et devenait petit à petit plus urbanisée et relativement industrialisée. La concentration de la population s'opérait dans quelques points géographiques des quelque deux millions de kilomètres carrés de la superficie du pays, là où la plupart des services publics se concentraient. Le processus d'urbanisation a modifié les habitudes culturelles de la population ; l'éducation, quoique encore précaire, a atteint des sommets jamais vus sur le territoire mexicain. Le Mexique est entré dans une phase de stabilisation politique, état qu'il n'avait jamais connu pendant toute son existence comme pays indépendant.

\section{L'église et l'organisation de sa présence dans l'espace public}

21 L'histoire des conflits politico-religieux entre l'Église et l'État montre que les conditions n'étaient pas favorables à un développement harmonieux entre ces deux groupes, ni à la mise sur pied de mécanismes de communication efficaces; et le problème se posait de façon plus aiguë pour la hiérarchie catholique mexicaine qui se trouvait désavantagée par rapport à ce qui se passait ailleurs en Amérique latine. Déjà, dans les années quarante et cinquante, l'Église avait pu mettre en place, par exemple, des radios éducatives en Colombie et des radios minières en Bolivie. Ces deux expériences faisaient partie de stratégies d'éducation et de communication régionale élaborées par le clergé qui pouvait agir soit comme administrateurs des médias, soit comme propriétaires, tout en jouissant en même temps d'importants appuis de l'État (Beltrán, $1994: 73,74,75$ ), ce qui était interdit au clergé mexicain à qui on refusait toujours toute participation directe à la gestion des médias.

Communication et organisation, 9 | 1996 

vigueur. Il s'agit d'un amendement à l'article 130 dans lequel est reconnue la séparation entre l'État et les Églises. Bien que la séparation entre les deux institutions existait de fait, ce n'est qu'à partir de 1992 que cette séparation a acquis un caractère légal. Moreno Padilla signale que :

« au premier paragraphe (de l'article 130 de la constitution mexicaine) on réaffirme et on consacre de façon expresse le principe de la séparation entre l'État et les Eglises. Ce paragraphe-là n'existait pas d'une manière explicite auparavant car jusqu'à ce nouveau texte constitutionnel, les Églises n'avaient pas de personnalité juridique » (Moreno, 1995 :181).

Il est évident que l'absence de caractère juridique a Privé les Églises et, particulièrement, la catholique, de liberté de manœuvre. Le culte et le développement des activités propres au culte ont été confinés à l'intérieur des temples ainsi que des enceintes particulièrement établies Pour la pratique des religions. 
«tout homme est libre de professer la croyance religieuse qu'il juge la meilleure, ainsi que de pratiquer les cérémonies, dévotions ou actes de culte qui s'y rapportent, à condition que cela n'implique pas de délits ou de fautes contraires à la loi.»

«Le Parlement ne peut dicter de lois qui établissent ou interdisent une religion quelconque. »

"Les actes religieux publics de culte se tiendront en temps normal dans les temples. Ceux qui, de façon extraordinaire, auraient lieu en dehors de ceux-ci, se tiendront conformément aux dispositions des lois et règlements » (Article 24 de la Constitution politique Mexicaine). de la hiérarchie religieuse, principalement et quasiment de façon exclusive avec la catholique, qui ont abouti à la modification de l'article 130 où explicitement la personnalité juridique des Églises était reconnue avec cependant, il faut le souligner, de nombreuses restrictions à plusieurs niveaux. l'obligeait à agir prudemment et à modifier des attitudes gouvernementales archaïques et contraires à l'esprit de l'ouverture internationale comme l'étaient les structures protectionistes au niveau économique et corporatistes au niveau social. Blancarte signale que les réactions des Églises à ces changements ont été immédiates mais non homogènes :

« de cette manière, il a été mis en évidence que l'attitude de l'Église et en particulier celle de l'Épiscopat n'a pas été de se soumettre aux directives gouvernementales, malgré leur probable reconnaissance envers le président pour les réformes faites dans le domaine religieux. La hiérarchie catholique a perçu l'étroit rapport entre les libertés politiques et les libertés religieuses; c'est-à-dire, l'Église allait maintenant tirer profit de l'instauration d'un régime authentiquement démocratique au Mexique » (Blancarte, 1995 :57,58). 


\section{L'église et l'éducation : une voie détournée}

Dans le contexte mexicain des trente dernières années, le champ de l'éducation est devenu pour l'Église catholique, une voie détournée d'appropriation de pouvoir.

En 1929, le pape Pie XI rendait publique l'encyclique Divini Illius Magistri dont l'idée centrale spécifiait que l'éducation devait désormais faire partie des préoccupations premières de l'Église. C'est ainsi que, lentement, la confrontation entre l'État et l'Église mexicains va se déplacer sur le terrain éducatif. Les partisans du processus de sécularisation de la culture nationale estimaient que « le concept de laïcité n'avait pas fait son travail, en devenant Plus large et plus vaste que le concept de religion, parce que même s'il ne niait pas la religion, il ne l'incluait pas non plus » (Negrete, 1988 :155).

Sur le terrain éducatif, l'Église est passée dans une semi-clandestinité, dans la mesure où la Constitution prévoyait de fortes limitations à l'éducation religieuse ou plus exactement, à la participation de l'Église catholique dans l'administration de l'enseignement. Néanmoins, face à l'augmentation de la pression de la population catholique, l'État a établi quelques mesures où il assurait la validité de l'éducation laïque tout en acceptant l'éducation religieuse, mais sans lui assurer une reconnaissance officielle.

La confrontation sur le terrain éducatif s'est intensifiée. L'État a formulé des prescriptions pour les institutions privées :

a) Enseignement laï.

b) Méthodes et contenus pédagogiques.

c) Enseignants laïcs.

d) Financement éducatif laïc (Negrete, $1988: 161)$.

Ces dispositions ont fait réagir vivement l'Église et les associations de parents, ce qui a provoqué de forts affrontements sur le terrain idéologique. D'importantes opérations de propagande se sont développées contre l'instruction que l'État prétendait implanter. La pression sociale des principaux groupes conservateurs a fini par obtenir une modification à la loi de l'éducation en 1946 (Iturriaga de la Fuente : 1951/1994).

Durant ces années, les fronts de la reconnaissance de la personnalité juridique des religions et de l'accès à la propriété et à la gestion des mass-médias ont connu une sorte de pause dans les tensions entre les deux parties (l'Église et l'État) ; l'activité éducative est devenue, elle, un terrain très actif.

Tout d'abord, l'Église catholique mexicaine a montré un intérêt spécial pour la thématique de la réaffirmation des cultures régionales comme le signale White, «plusieurs porte-parole [sont entrés] dans une sorte d'alliance avec les mouvements populaires contre la nouvelle culture transnationale qui pénètre en Amérique latine et, avec eux, des symboles socio-théologiques se sont développés qui projettent un autre modèle de société » (White, 1987 :219).

Le champ éducatif a été tout au long de l'histoire du Mexique, un domaine fondamental pour les activités de l'église catholique. Faut-il s'étonner d'observer le fait que les différents départements des Sciences de l'information et de la communication sociale appartiennent ou sont administrés par des groupes religieux, la plupart d'entre eux catholiques. Parmi ceux-ci, il faut remarquer les Jésuites. 
48 L'une des préoccupations de ces écoles, en ce qui a trait à l'enseignement de la communication, a été non pas l'instruction confessionnelle mais l'application des valeurs déontologiques dans les métiers de la communication : la publicité, le journalisme, les relations publiques. Par exemple, l'enseignement d'une éthique professionnelle a été l'un des aspects auquel on a consacré le plus d'attention dans l'enseignement de la publicité (White, 1987).

49 Par ailleurs, il faut remarquer que la science de la communication est relativement récente dans le champ de l'éducation au Mexique. Les premières écoles des sciences de la communication se sont établies dans les années 60 et il faut attirer l'attention sur la Universidad Iberoamericana (jésuite) considérée comme pionnière dans cette branche (Fuentes, 1991).

50 Le terrain éducatif a constitué, d'autre part, un élément de liaison entre la communauté et les universités religieuses, dans la mesure où l'une des dispositions du système éducatif mexicain impose la prestation d'un service communautaire. Celui-ci constitue un véritable facteur de liaison entre les universités et les communautés parce que les étudiants font des stages professionnels dans les communautés. Ainsi, celles-ci en viennent à disposer de conseillers qualifiés dans la personne de ces étudiants-stagiaires. $\mathrm{Au}$ fil des ans, ce rapprochement communautaire a constitué le principal mécanisme de participation régionale de nombreuses organisations religieuses.

\section{Sécuralisation de la société}

51 L'intégration des structures institutionnelles de l'État marquée par un fort centralisme s'opposait aux structures régionales mises en place par l'Église. Les espaces nationaux du pouvoir étaient dirigés par l'État mais l'Église, par sa présence auprès de la population, se proposait d'occuper le niveau régional. Il y avait deux importantes raisons pour qu'elle privilégie cette direction. D'abord, le développement des sociétés protestantes qui élargissaient leur présence au niveau régional. D'autre part, l'espace régional était négligé par l'organisation centralisatrice de l'État.

52 Une partie du succès de l'enracinement des groupes religieux dans les communautés régionales s'explique par leur approche et leur utilisation de techniques d'intégration sociale telles que l'enseignement primaire et les publications à circulation régionale, utilisant le langage et les symboles propres aux communautés dans lesquelles ils travaillaient.

53 Les travaux de communication communautaire avaient comme base, dans plusieurs cas, l'encyclique Rerum Novarum dans la mesure où celle-ci affirmait que toutes les actions devaient se considérer comme un acte de justice et de charité sociale et non pas comme le résultat de la lutte de classes. De cette façon, on suivait les principes sociaux de l'Église (Negrete, $1988: 249$ ).

\section{Nouvelles réactions de l'église}

54 Dès les années 70, l'État a adopté, toute proportion gardée, une attitude beaucoup plus tolérante face aux cultes religieux pendant que l'Église catholique, de son côté, entamait un processus de sécularisation de l'organisation religieuse. 

de sa logique constitutionnelle relative à l'éducation; ils ont cependant provoqué, de façon irrégulière mais croissante, une sorte de validation de l'éducation offerte par les institutions privées. a présenté de nouvlles stratégies d'action. Le domain éducatif acquérait ainsi un intérêt fondamental. Mais il fallait aussi rejoindre la population non touchée par l'éducation. L'un des spécialistes de l'histoire du catholicisme au Mexique signale que l'Église s'est mise à appuyer les laïcs militants catholiques en raison de leur meilleure pénétration du milieu laïc. Bien sûr, cela a constitué un mouvement qui était « contrôlé et coordonné par les autorités religieuses et, par conséquent, conforme aux doctrines et principes de l'Église » (Negrete, 1988 :241).

57 En dépit de la force que le mouvement en faveur de l'Église avait obtenu, les espoirs de l'Église n'étaient pas Pleinement comblés, peut être parce que, comme le dit le sociologue mexicain Morán, « l'État libéral a été capable de prévoir les conséquences et le prix de son projet [d'État] ainsi que des projets «alternatifs », même peu définis » (Morán et Bravo, 1989 :51).

58 L'Église a maintenu ses positions avec une force relative, comme le signale Bastian, car elle a «été historiquement l'unique institution capable de forger l'unité nationale pardessus tous les nationalismes et par-dessus toutes les identités éthniques atomisées » (Bastian, 1990 :24).

\section{Le rôle des bureaux de communication sociale dans les archevêchés}

59 Malgré tous les changements qui s'opéraient dans le contexte politique des rapports Église-État, il est quand même nécessaire de signaler le fait qu'au sein de la structure même de l'Église catholique, dès les années 60 , ont commencé à se manifester des tendances divergentes qui concernaient, d'une part, ses formes d'organisation et, d'autre part, sa fonction sociale.

60 Les différentes perspectives d'organisation de l'Église ont débouché sur deux fronts d'action dans le processus de modernisation : a) à l'externe se posait le conflit Église-État et, b) à l'interne s'affrontaient les diverses tendances touchant la «théologie de la libération ».

61 Pendant les années 60 et 70, différentes actions, surtout à caractère politique, ont été engagées par des membres de la communauté religieuse catholique à travers le Mexique, mais elles ne répondaient pas à une stratégie nationale d'action concertée. Ces événements ont fait apparaître le manque de coordination entre les principaux dirigeants et sont à l'origine d'une restructuration interne de la communauté catholique.

62 Ce sont de nouveaux gestionnaires qui vont réformer les structures administratives de l'Église sur le modèle d'une institution privée, c'est-à-dire d'une « organisation ».

63 Pendant les années 60 et avec de nombreuses modifications au départ, le Secrétariat Social Mexicain - Secretariado Social Mexicano - s'est formé. Ce Secrétariat est une organisation centrale pour la coordination ecclésiastique. Cet organisme a été à l'origine de la formation de bien d'autres institutions, dont :

Communication et organisation, 9 | 1996 
a) L'Union de l'Aide Mutuelle de l'Épiscopat (UMAE)

b) La Société Théologique Mexicaine (STM)

c) Le Centre National de la Communication Sociale (CENCOS)

d) Le Centre de Recherche et d'Action Sociale (CIAS)

e) Le Centre inter-culturel de Documentation (CIDOC)

La création de ces organismes-là a obéi à une stratégie d'organisation interne de l'Église catholique. Victor Muro signale que :

«Cette action à la base essaye de dessiner ce que l'on a appelé la Pastorale d'ensemble - Pastoral de Conjunto - qui en fin de compte, avait comme but de: coordonner les actions pastorales dans tout le pays selon les dispositions du Concile; rendre équitable la distribution des revenus aux clergés et développer la participation des laïcs dans toutes les activités ecclésiastiques.

La Pastorale d'ensemble amenait par conséquent à postuler un compromis social pour ses membres car le Concile imposait très clairement une action chrétienne qui devait incarner la réalité socio politique de la région » (Muro, 1995 :185).

70 Ces organes de communication sociale ont été créés pour toucher les structures internes de l'Église. Les différentes actions entamées par l'Église prenant une grande dimension politique, rendaient nécessaire l'instauration d'un organisme capable d'établir les fonctions de médiateur et de porte-parole.

71 Au cours des années 60 et 70, il y avait un fort besoin de structures de communication interne afin de concilier les perspectives ecclésiastiques et politiques des différents archevêchés du pays, cependant, CENCOS n'avait pas la Possibilité de devenir administrateur des mass-médias pour la simple raison que l'Église n'avait pas de personnalité juridique.

72 Cet empêchement juridique constituait un obstacle important. De plus, les blocages ne s'arrêtaient pas là, l'activité du prosélytisme religieux était aussi restreinte et le culte ne pouvait s'exercer que dans les temples selon ce que déterminait la Constitution dans son article 24 qui stipulait que les Églises n'avaient pas le droit d'utiliser d'autres espaces publics que les temples pour faire la promotion de leur idéologie religieuse. La combinaison de ces deux facteurs bloquait toute initiative sur ce terrain.

73 Les raisons qui ont motivé la création du CENCOS ne visaient donc pas pas forcément les mass-médias mais plutôt l'organisation d'une structure interne capable d'établir un réseau de communication pour essayer de résoudre les conflits existant à ce moment là au sein de l'organisation.

74 On peut signaler les cas des archevêchés du nord et du sud du pays dans lesquels l'activisme politique pendant les années 70 et 80 s'est manifesté de manière claire et directe et où l'intervention des hautes sphères de décision a été nécessaire obligeant, dans une certaine mesure, le CENCOS à devenir un porte-parole assez privilégié.

Les dispositions du Concile Vatican II où était signalée l'importance de l'usage des médias et plus particulièrement dans le Décret du Concile Vatican II Inter mirifica ainsi que dans les Instructions pastorales du Conseil Pontifical pour les Communications Sociales Comuni et Pregressio et Aetatis novæ, ont été effectivement prises en compte au Mexique mais seulement après 1992, année où les rapports politico-légaux ont changé. Mais pas suffisamment pour que les organismes tels que le CENCOS arrivent à avoir sous sa tutelle le contrôle d'un mass-média. Des essais récents ont été effectués en utilisant des stratégies employées par des cultes protestants des États Unis, qui ont réussi à intervenir 
dans les chaînes de radio. Cependant, il est encore trop tôt pour tirer les conclusions de ces expériences.

\section{En guise de conclusion}

\section{catholique et l'État mexicain témoignent d'une évolution politique particulière qui met en évidence, de façon fondamentale, une lutte pour le contrôle hégémonique de l'espace public.}

77 La reprise des rapports politiques entre les deux institutions a commencé très tardivement par rapport aux autres pays de l'Amérique latine. La plupart des études portant sur ce sujet mettent l'accent sur le contenu des rapports politiques entre l'Église (notamment catholique) et l'État. D'autre part, peu d'études abordent une réflexion sur l'évolution de la situation actuelle et future des rapports Église-État au Mexique. Dans la « déclaration des évêques mexicains sur la nouvelle « Loi des Associations religieuses et le culte public " ", on peut lire que :

«personne ne doit se surprendre de l'intérêt des évêques pour cette loi parce que l'Église, comme la réalité complexe qu'elle est, composée par des éléments divins et humains, n'est pas seulement attachée à la loi de Dieu et à ses propres lois mais elle doit agir dans le cadre juridique de la société civile » (Garcia Ugarte, 1993 :293).

Les relations s'exercent maintenant dans un nouveau Pacte d'organisation politique de l'État. L'accès aux mass-médias de la part de l'Église catholique est récent. Il n'existe donc presque pas de bibliographie concernant ce sujet. La participation de l'Église catholique dans le panorama médiatique commence à devenir un fait actuel mais pas dans tout le Mexique. C'est ce qui rend difficile toute recherche en profondeur sur ce sujet.

79 À n'en pas douter, l'ouverture de l'État envers les religions pourra avoir comme conséquence moins d'ambiguïté dans leurs rapports ainsi qu'un changement politique d'attitude entre les classes politiques mexicaines et les secteurs religieux.

«Actas y documentos pontificios (1992) : Uso de los instrumentos de comunicación social en la promoción de la fé. Mexique.Ediciones Paulinas.

81 - CELAM (1987) : Comunicación : misión y desafío. Bogotá (Colombie). Ediciones Paulinas.

82 - Jalisco a tiempo (1994) : Primer informe sobre problemas del desarrollo y alternativas de políticas públicas. Guadalajara (Mexique). Universidad de Guadalajara.

83 - Journaux : El Norte de Monterrey (Mexique). La Jornada

84 - Revue : Proceso 


\section{BIBLIOGRAPHIE}

BASTIAN, Jean-Pierre (1990) : Heterodoxia religiosa y cambio social. El impacto regional de las sociedades no católicas en México. Paris, p. 23-42. In Rodolfo Morán (Comp.) La Política y el cielo. Guadalajara (Mexique). Universidad de Guadalajara.

BELTRAN SALMON, Luis Remiro (1994) : Neoliberalismo y comunicación democrática en latinoamérica : «Plataformas y banderas » para el tercer milenio. In Nuevos rostros. Quito (Ecuateur). OCIC. Latinoamérica, UNDAD-AL y UCLAP.

BLANCARTE, Roberto (1993) : Modernidad, secularización y religión; la iglesia católica, el estado y las sociedad mexicana en el umbral del siglo XXI, p. 152-188. In México a fines de siglo, Tomo II. Mexique. Fondo de Cultura Económica et Consejo Nacional para la Cultura y las Artes.

(1995) : La doctrina social católica ante la democracia moderna, pp. 19-58. In Religión, iglesias y democracia. Mexique. La Jornada Ediciones et CIIH/UNAM.

FUENTES NAVARRO, Raúl (1991) : La comunidad desapercibida. Guadalajara (Mexique). ITESOCONEICC.

GARCIA UGARTE, María Eugenia (1993) : La nueva relación Iglesia-Estado en México. Mexique. Nueva Imagen.

MONSIVAIS, Carlos (1993) : Los espacios de las masas pp. 267-308. In México a fines de siglo, Tomo I. Mexique. Fondo de Cultura Económica et Consejo Nacional para la Cultura y las Artes.

MORAN, Rodolfet Patricia BRAVO (1989) : Doctrina religiosa y escuelas particulares. Cuadernos de Divulgación (II Epoca) N² 29. Guadalajara (mexique). Universidad de Guadalajara.

MURO, Víctor Gabriel (1990) : El factor religioso en los movimientos populares. In Rodolfo Morán (Comp.) La política y el cielo. Guadalajara (Mexique). Universidad de Guadalajara.

(1995) : Iglesia, movimientos sociales y democracia P.p. 181 -200. In Religión, iglesias y democracia. Mexique. La Jornada Ediciones et CIIH/UNAM.

NEGRETE, Martaelena (1988) : Relaciones entre la Iglesia y el Estado en México, 1930-1940.

México, D.F. Colegio de México et Universidad Iberoamericana

RAMOS CORTES, Víctor M. (1992) : Poder, representación y pluralidad en la Iglesia. Guadalajara (Mexique). Universidad de Guadalajara.

WHITE, Robert A. (1987) : Iglesia y publicidad en América latina pp. 213-255. In IPAL : Publicidad, la otra cultura. Lima (Perú). Instituto para América latina.

\section{RÉSUMÉS}

Jusqu'en 1992, la constitution du Mexique niait toute personnalité juridique à l'église et encadrait les activités de celle-ci de façon très serrée. C'est ainsi que l'Église ne pouvait avoir accès à la propriété des médias. Pour occuper l'espace public, l'Église catholique adopta une double stratégie : elle choisit d'investir d'une part le champ de l'éducation et fonda les premières Écoles 
de communication; d'autre part, elle se rapprocha de la base en développant des actions de support à la collectivité, comme en témoigne l'engagement de Mgr Ruiz dans le Chiapas.

Until 1992, the Mexican Constitution denied any juridical personality to the Church and surrounded its activities in a very tight manner. Thus, the Church could not have access to media property. To retain public space, the Catholic Church adopted a twofold strategy: on the one hand, she chose to invest in the field of education; and founded the first communication schools; on the other hand, she strove to draw closer to the base by develo-ping supportive actions for the community, as witness the involvement of Mgr Ruiz in the Chiapas.

\section{AUTEUR}

\section{ARMANDO ZACARÍAS CASTILLO}

Armando Zacarias Castillo est professeur et chercheur à l'Université de Guadalajara, au Mexique. Il a publié divers articles sur le journalisme mexicain dont La prensa en México : une mirada a su historia, dans Presse et médias au Mexique, in Mondes Hispanophones 24, Presses de l'Université de Rennes. M. Castillo est responsable de la Revista Universidad de Guadalajara. 